SECTION 2. Applied mathematics. Mathematical modeling.

Sizova S.A. student

Stavropol State agrarian University

Murdugova V.Yu. student

Stavropol State agrarian University

Meleshko Svetlana Vasilyevna assistant of the Department of mathematics

Stavropol State agrarian University

\title{
LINEAR PROGRAMMING AS A DOMAIN OF MATHEMATICAL PROGRAMMING IN ECONOMIC CHALLENGES
}

Of linear programming in the economy.

Keywords: Economics, programming.

\section{ЛИНЕЙНОЕ ПРОГРАММИРОВАНИЕ КАК ОБЛАСТЬ МАТЕМАТИЧЕСКОГО ПРОГРАММИРОВАНИЯ ПРИ РЕШЕНИИ ЭКОНОМИЧЕСКИХ ЗАДАЧ}

О линейном программировании в экономике.

Ключевые слова: экономика, программирование.

Математические изучения отдельных экономических проблем, математическая формализация числового материала проводилась ещё в XIX веке. При математическом анализе процесса расширенного производства использовались алгебраические соотношения, анализ которых проводился с помощью дифференциального исчисления. Это давало вероятность получить всеобщее представление о проблеме.

Совершенствование экономики потребовало количественных показателей, и в 1920 годы был создан межотраслевой баланс, который послужил толчком в деле создания и исследования математических моделей. Разработка межотраслевого баланса в 1924-1925 годах в СССР повлияла на работы экономиста и статистика Василия Васильевича Леонтьева, который разработал межотраслевую модель производства и распределения продукции. В 1938 году Леонид Витальевич Канторович в порядке научной консультации приступил к изучению чисто практической задачи по составлению наилучшего плана загрузки лущильных станков 
(фанерный трест). Эта задача не поддавалась обычным методам потому, что она была не случайная.

В 1939 году Канторович опубликовал работу «Математические методы организации и планирования производства», в которой сформулировал новый класс экстремальных задач с ограничениями и разработал эффективный метод их решения. Таким образом были заложены основы линейного программирования [4].

Изучение подобных задач привело к созданию новой научной дисциплины - линейного программирования и открыло новый этап в развитии экономико-математических методов.

Термин «программирование» понимается как «планирование» (один из переводов англ. programming). Он был предложен в середине 1940-х годов одним из основателей линейного программирования Джорджем Данцигом, ещё до того, как компьютеры были использованы для решения линейных задач оптимизации.

Линейное программирование - область математического программирования, посвященная теории и методам решения экстремальных задач, характеризующихся линейной зависимостью между переменными.

В самом общем виде задачу линейного программирования можно записать так. Даны ограничения типа:

$$
\begin{gathered}
\sum_{j=1}^{n} a_{i j} x_{j} \leq b_{i}, i=1,2, \ldots, m_{1}, \\
\sum_{j=1}^{n} a_{i j} x_{j}=b_{i}, i=m_{1}+1, m_{1}+2, \ldots, m_{2}, \\
\sum_{j=1}^{n} a_{i j} x_{j} \geq b_{i}, i=m_{2}+1, m_{2}+2, \ldots, m,
\end{gathered}
$$

или в так называемой канонической форме, к которой можно привести все три указанных случая:

$$
\sum_{j=1}^{n} a_{i j} x_{j}=b_{i}, i=1,2, \ldots, m
$$

Требуется найти неотрицательные числа $x_{j}(j=1,2, \ldots, n)$, которые минимизируют (или максимизируют) линейную форму

$$
Z=\sum_{j=1}^{n} c_{j} x_{j},
$$

Неотрицательность искомых чисел записывается так: $x_{j} \geq 0$. 
Таким образом, здесь показана общая задача математического программирования с оговорками: как ограничения, так и целевая функция линейные, а искомые переменные неотрицательные. Обозначения можно трактовать следующим образом: $b_{i}$ - количество ресурса вида $i ; m-$ количество видов этих ресурсов; $a_{i j}$ - норма расхода ресурса вида $i$ на единицу продукции вида $j ; x_{j}$ - количество продукции вида $j$, причем количество таких видов $-n ; c_{j}$ - доход (или другой выигрыш) от единицы этой продукции, а в случае задачи на минимум - затраты на единицу продукции $[4,6]$. Нумерация ресурсов разделена на три части: от 1 до $m_{1}$, от $m_{1}+1$ до $m_{2}$ и от $m_{1}+2$ до $m$ в зависимости от того, какие ставятся ограничения на расходование этих ресурсов. В первом случае «не больше», во втором - «столько же», в третьем - «не меньше». $Z$ - в случае максимизации, например, объем дохода или продукции, в случае же минимизации - расход сырья, себестоимость и тому подобное.

Понятие “программирование” объясняется здесь тем, что в процессе решения задачи обнаруживаются неизвестные переменные, которые, обычно в совокупности предопределяют программу или план работы какого-либо экономического объекта. Факт линейной зависимости между переменными выражает слово “линейное". Причем задача обязательно имеет экстремальный характер, то есть. состоит в отыскании экстремума (максимума или минимума) целевой функции.

Следует отметить: так как в реальной экономике подавляющее большинство зависимостей носит более сложный нелинейный характер, то предпосылка линейности - есть огрубление, упрощение действительности. В одних случаях оно достаточно реалистично, в других же выводы, которые получаются с помощью решения задач линейного программирования, являются весьма несовершенными. примере.

Разберем построение задачи линейного программирования на

Продукцией городского молочного завода являются молоко, кефир и сметана, расфасованные в бутылки. На производство 1 т молока, кефира и сметаны требуется соответственно 1100, 1100 и 8700 кг молока. При этом затраты рабочего времени при разливе 1 т молока и кефира составляют 0,15 и 0,17 машино-часов. На расфасовке 1 т сметаны заняты специальные автоматы в течение 4,10 часов. Всего для производства цельномолочной продукции завод может использовать 275000 кг молока. Основное оборудование может быть занято в течение 19,5 машино-часов, а автоматы по расфасовке сметаны - в течение 20,25 часов. Прибыль от реализации $1 \mathrm{~T}$ молока, кефира и сметаны соответственно равна 40, 32 и 149 руб. Завод должен ежедневно производить не менее 100 т молока, расфасованного в 
бутылки. На производство другой продукции не имеется никаких ограничений.

Требуется определить, какую продукцию и в каком количестве следует ежедневно изготовлять заводу, чтобы прибыль от ее реализации была максимальной. Составить математическую модель задачи.

Решение. Предположим, что молочный завод будет ежедневно производить $x_{1}$ тонн молока, $x_{2}$ тонн кефира и $x_{3}$ тонн сметаны. Тогда ему для изготовления этой продукции необходимо $1100 x_{1}+1100 x_{2}+8700 x_{3}$ кг молока.

Так как завод может использовать ежедневно не более 275000 кг молока, то должно выполняться неравенство

$$
1100 x_{1}+1100 x_{2}+8700 x_{3} \leq 275000
$$

Аналогичные рассуждения, проведенные относительно возможного использования линий разлива цельномолочной продукции и автоматов по расфасовке сметаны, позволяют записать следующие неравенства:

$$
\begin{gathered}
0,15 x_{1}+0,17 x_{2} \leq 19,5, \\
4,10 x_{2} \leq 20,25
\end{gathered}
$$

Так как ежедневно должно вырабатываться не менее 100 т молока, то $x_{1} \geq 100$. Далее, по своему экономическому смыслу переменные $x_{1}$ и $x_{2}$ могут принимать только лишь неотрицательные значения: $x_{2} \geq 0, x_{3} \geq 0$. Общая прибыль от реализации $x_{1}$ тонн молока, $x_{2}$ тонн кефира и $x_{3}$ тонн сметаны равна $40 x_{1}+32 x_{2}+149 x_{3}$ руб. Таким образом, приходим к следующей математической задаче. Дана система

$$
\left\{\begin{array}{l}
1100 x_{1}+1100 x_{2}+8700 x_{3} \leq 275000 \\
0,15 x_{1}+0,17 x_{2} \leq 19,5 \\
4,10 x_{2} \leq 20,25 \\
x_{1} \geq 100
\end{array}\right.
$$

четырех линейных неравенств с тремя неизвестными $x_{1}, x_{2}, x_{3}$ и линейная функция относительно этих же переменных

$$
F=40 x_{1}+32 x_{2}+149 x_{3}
$$

Требуется среди всех неотрицательных решений системы неравенств (1) найти такое, при котором функция (2) принимает максимальное значение. Так как система (1) представляет собой совокупность линейных неравенств и функция (2) линейная, то исходная задача является задачей линейного программирования.

При решении задач линейного программирования можно выделить следующие этапы построения:

- определение цели, то есть чего необходимо добиться, решая задачу; 
- определение параметров модели, то есть заранее известных фиксированных факторов, на значения которых исследователь не влияет;

- формирование управляющих переменных, изменяя значение которых можно приближаться к поставленной цели. Эти значения и являются решениями задачи;

- определение области допустимых решений, т.е. тех ограничений, которым должны удовлетворять управляющие переменные;

- выявление неизвестных факторов, т.е. величин, которые могут изменяться случайным или неопределенным образом.

Математические методы являются важнейшим инструментом анализа экономических явлений и процессов, построения теоретических моделей, позволяющих отобразить существующие связи в экономической жизни, прогнозировать поведение экономических субъектов и экономическую динамику. Они ускоряют проведение экономического анализа, способствуют более полному учету влияния факторов на результаты деятельности и повышению точности вычислений.

\section{Литература}

1. Акулич И.Л. Математическое программирование в примерах и задачах / И.Л. Акулич - М.: Высшая школа - 1986 - 319 с.

2. Бодров В.И. Математические методы принятия решений Учебное пособие / В.И. Бодров, Т.Я. Лазарева, Ю.Ф. Мартемьянов - Тамбов - 2004. $-124 \mathrm{c}$.

3. Гельман, В.Я. Решение математических задач средствами Excel: Практикум / В.Я. Гельман. - СПб.: Питер - 2003. - 237 с.

4. Ермаков В.И. Общий курс высшей математики для экономистов: Учебник. - М.: ИНФРА-М, 2002.- 656с.

5. Коршунова Н.И. Математика в экономике. Учебное пособие/ Н.И. Корщунова, В.С. Пласунов - М.: Вита-Пресс - 1996. - 368 с.

6. Красс М.С. Основы математики и ее приложения в экономическом анализе. Учебник-3-е изд., исп. / М.С. Красс, Б.П. Чупрынов -М.: Дело, 2002. $-688 \mathrm{c}$. 